\title{
Time-delayed feedback control of diffusion in random walkers
}

\author{
Hiroyasu Ando, ${ }^{1}$ Kohta Takehara, ${ }^{2}$ and Miki U. Kobayashi ${ }^{3}$ \\ ${ }^{1}$ Faculty of Engineering, Information and Systems, University of Tsukuba, 1-1-1 Ten-noudai, Tsukuba 305-8573 Japan \\ ${ }^{2}$ Department of Business Administration, Graduate School of Social Science, Tokyo Metropolitan University, 1-4-1 Marunouchi, \\ Chiyoda-ku, Tokyo 100-0005, Japan \\ ${ }^{3}$ Faculty of Economics, Rissho University, 4-2-16 Osaki, Shinagawa-ku, Tokyo 141-8602, Japan
}

(Received 23 January 2017; published 25 July 2017)

\begin{abstract}
Time delay in general leads to instability in some systems, while specific feedback with delay can control fluctuated motion in nonlinear deterministic systems to a stable state. In this paper, we consider a stochastic process, i.e., a random walk, and observe its diffusion phenomenon with time-delayed feedback. As a result, the diffusion coefficient decreases with increasing delay time. We analytically illustrate this suppression of diffusion by using stochastic delay differential equations and justify the feasibility of this suppression by applying time-delayed feedback to a molecular dynamics model.
\end{abstract}

DOI: 10.1103/PhysRevE.96.012148

\section{INTRODUCTION}

Diffusion phenomena are ubiquitous in nature, e.g., in Brownian motion [1], biological membranes [2,3], fluid systems [4], engineering systems [5], material science [6], and so on. These diffusion phenomena are sources of noise and are unavoidable in experimental systems [7,8]. Noise can prevent the precise manipulation of small scale systems. However, it can also be helpful in engineering a system by using physical phenomena such as logical stochastic resonance, where a certain amount of noise is required for logical operations $[9,10]$. In both cases, it is important to control noise in terms of the variance of stochastic processes, because noise is normally adjusted according to its strength. If the variance of noise is controlled, it would be useful for various kinds of applications.

When we consider systems with noise from a theoretical viewpoint, mathematical models are convenient, because they can numerically simulate an experiment. A mathematical model of Brownian motion is a typical example of a noisy system, which can be modeled by a simple stochastic description, such as the Langevin equation. For practical purposes, it is important to consider how diffusion can be suppressed in the Brownian motion model. The most commonly used approach for suppressing diffusion is to decrease the temperature of the whole system, resulting in a suppression of thermal fluctuations, namely, the relation $D \sim \mathbf{T}$, where $D$ is the diffusion coefficient and $\mathbf{T}$ is the temperature. However, the temperature is decreased for all elements in the system and is not selective for specific targets. Therefore, such a control is expensive. We introduce an alternative method that can control the diffusion processes of one particle in Brownian motion.

In terms of cooling nanomicroscopic systems, a mathematical model of feedback cooling in electromechanical oscillators [11] and a generalized model of Langevin dynamics with non-Markovian feedback [12] have been explored. In these systems, time delay in the feedback loop is inevitable due to a time lag between the sensor and the manipulator. Furthermore, in nonlinear systems, a time-delayed feedback control (DFC) method has been proposed to stabilize regular motion in fluctuated dynamics [13]. In general, time delay leads to system instability. However, DFC proves that time delay can stabilize deterministic systems. On the other hand, in stochastic systems, e.g., a random walk model with time delay, destabilization has been observed as a result of delay [14]. In addition, a coherent noise-induced oscillation is observed in the van der Pol system with delayed feedback [15]. Thus, we consider whether DFC can control the amount of diffusion in a random walk.

In this paper, we focus on the stochastic diffusion of random walks, corresponding to Brownian motion in discrete time, and apply the DFC method to control the extent of this diffusion. First, we observe diffusion processes in a one-dimensional (1D) random walk model, controlled by the DFC method, and analyze control in terms of stochastic delay differential equations. Second, we apply the method to a molecular dynamics model of two flocculated particles with thermal fluctuations that interact via the Lennard-Jones (LJ) potential. We numerically confirm that it is possible to suppress diffusion in the molecular dynamics model by the DFC method. It is noticed that, in conventional control theory, a single path is controlled to a certain state. In contrast, the purpose of this study is to statistically control stochastic processes.

\section{DELAYED FEEDBACK CONTROL OF RANDOM WALK}

In general, nonlinear functions with neutral fixed points show noise-induced diffusion phenomena. If these functions are linearized around the neutral fixed points, the following random walk model can be derived,

$$
x_{n+1}=x_{n}+D \xi_{n},
$$

where $x_{n}$ is a state variable for discrete time $n, \xi_{n}$ is an $m$ dimensional random variable following the normal distribution $N(0,1)$ for each entry, and $D$ is an amplitude vector of noise. Without loss of generality, we consider a 1D case, where $m=1$.

In order to control the random walk in $x_{n}$, we introduce a time-delayed feedback control method proposed by Pyragas [13] that is defined by the expression

$$
x_{n+1}=x_{n}+D \xi_{n}-K\left(x_{n}-x_{n-l}\right) .
$$

In Eq. (2), the DFC is applied to the model (1), where $K$ is feedback gain with a value of $0 \leqslant K<1$ and $l$ is the delay 
time. Any nonlinear function with noise can be linearized around its stable fixed point and described in the form of (2) as follows.

We consider the system

$$
x_{n+1}=f\left(x_{n}, a\right),
$$

where the map $f$ is nonlinear and has a stable or neutrally stable fixed point. $a$ is a parameter. We assume that the fixed point is denoted by $\tilde{x}$, and linearize $f$ around $\tilde{x}$. This results in

$$
x_{n+1}=f(\tilde{x})+f^{\prime}(\tilde{x})\left(x_{n}-\tilde{x}\right),
$$

which can be described as

$$
x_{n+1}=f^{\prime}(\tilde{x}) x_{n}+\left[1-f^{\prime}(\tilde{x})\right] \tilde{x},
$$

where we use the relation $\tilde{x}=f(\tilde{x})$. For this equation, if we denote $K=1-f^{\prime}(\tilde{x})$,

$$
x_{n+1}=(1-K) x_{n}+K \tilde{x} .
$$

Regarding this linear map, we add the noise term and rewrite (6) as

$$
x_{n+1}=x_{n}+D \xi_{n}-K\left(x_{n}-\tilde{x}\right) .
$$

The dynamics of the system (7) is simple, such that a trajectory fluctuates around the fixed point $\tilde{x}$. It is possible to suppress diffusion of a random walk by the form of (7), in which the diffusion coefficient is zero. On the other hand, in order to control a nonzero diffusion coefficient, we add the control term $K\left(x_{n-l}-\tilde{x}\right)$. Consequently, the system is in the form of DFC, namely, Eq. (2).

It should be noted that this discussion is relevant for systems with only stable fixed points and thus with no diffusion observed. By the procedure above, such stable systems are converted to systems showing diffusion because of the neutral fixed points.

Figure 1 shows the time series of the (2) system dynamics. It is observed that a longer $l$ suppresses the diffusion of a random walk. This result is counterintuitive, since a long time delay should make a system unstable. However, in the system (2), the opposite is observed. Thus, we quantify the diffusion decay with respect to $l$. In Fig. 2, the diffusion coefficients $\mathcal{D}$, defined by $\lim _{T \rightarrow T_{\infty}}\left\langle\left(X_{T}-X_{0}\right)^{2}\right\rangle / T$, are shown as a function

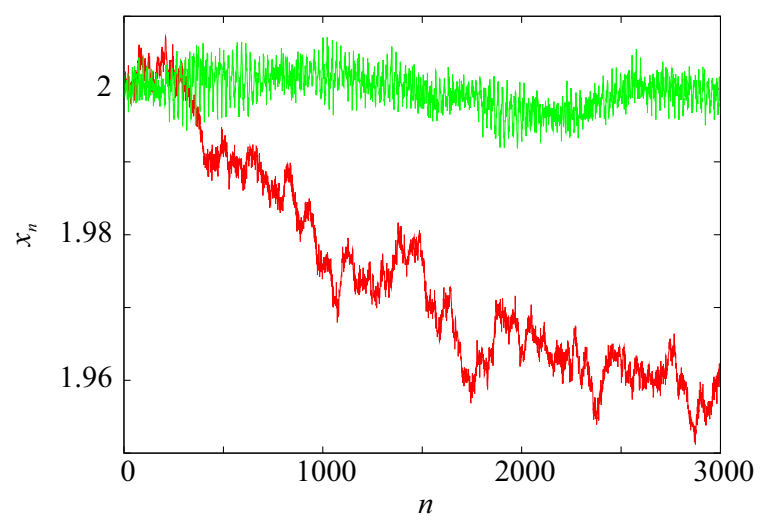

FIG. 1. Suppression of diffusion in random walks of the system (2). The red solid and green dashed lines represent when $l=2$ and $l=20$, respectively. $D=0.001$.

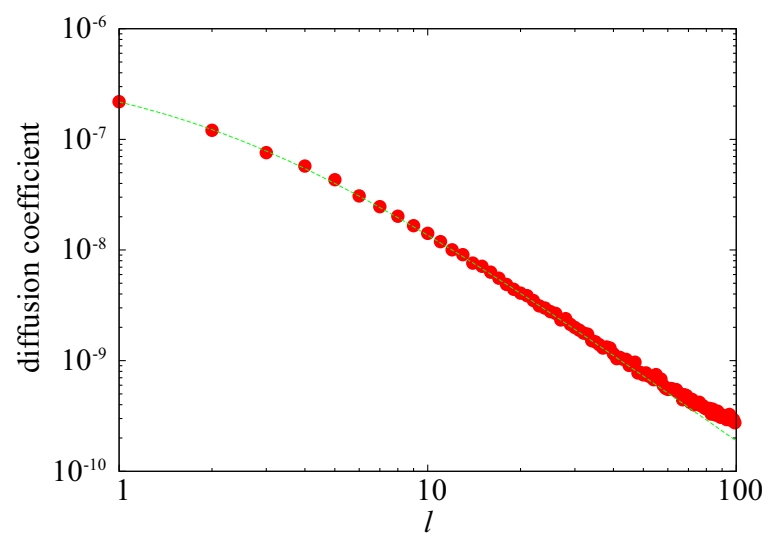

FIG. 2. $\mathcal{D}$ with respect to $l$, averaged over 1000 realizations. The dashed line is proportional to $1 /(1+K l)^{2} . K=0.5$.

of $l$. The result is averaged over 1000 realizations. We take $T_{\infty}=100000$. It is evident that $\mathcal{D}$ decays by $l^{-2}$. The decay order is illustrated in the following.

We consider a continuous time (2) system,

$$
\dot{y}_{t}=D \xi_{t}-K\left(y_{t}-y_{t-\tau}\right),
$$

where $y$ is a real variable and $t$ and $t-\tau$ are continuous time points. For this system, let $\Delta=\tau / l, l \in\{2,3, \ldots\}$. Then, we define $t_{n}$ as $n \Delta$, that is, $n \frac{\tau}{l}$, where $n \in\{-l,-l+$ $1, \ldots, 0,1, \ldots\}$. If we define $Y_{n}=y_{t_{n}}$ and $\xi_{n}=\xi_{t_{n}}$, Eq. (8) can be rewritten as $Y_{n+1}=Y_{n}+D \Delta \xi_{n}-\Delta K\left(Y_{n}-Y_{n-l}\right)$. This form is equivalent to Eq. (2).

We consider the continuous time system (8) using stochastic delay differential equations. In the standard setting of stochastic calculus, Eq. (8) is now redefined as

$$
d y_{t}=K\left(y_{t-\tau}-y_{t}\right) d t+D d w_{t}, \quad t \geqslant 0,
$$

where $\left\{w_{t}\right\}_{0 \leqslant t \leqslant T}$ is a standard Wiener process on a filtered probability space $\left(\Omega, \mathcal{F},\left\{\mathcal{F}_{t}\right\}, P\right)$, and $y_{s}$ for $s \in[\tau, 0]$ is assumed to be a given $\mathcal{F}_{0}$-measurable random variable $Z_{s}$. It should be noted that $\mathcal{F}_{t}$ contains all information for the realization of $w_{s}$ and $y_{s}$ for $s \leqslant t$. Thus, given $\mathcal{F}_{t}$, all of these variables can be all treated as constants. In the following, the method for determining the diffusion coefficient $\mathcal{D}$ is briefly described. For details, see the Appendix.

Equation (9) has the unique solution of

$$
\begin{aligned}
& y_{t}=y_{t}^{Z}+D \int_{0}^{t} y_{t-s}^{0} d w_{s}, \quad 0 \leqslant t, \\
& y_{t}=Z_{t}, \quad-\tau \leqslant t \leqslant 0,
\end{aligned}
$$

where

$$
\begin{aligned}
y_{t}^{Z} & :=y_{t}^{0} Z_{0}+K \int_{-\tau}^{0} y_{t-s-\tau}^{0} Z_{s} d s \\
y_{t}^{0}: & =\sum_{n=0}^{\lceil t / \tau\rceil}\left(\frac{K^{n}}{n !}\right)(t-n \tau)^{n} \exp [-K(t-n \tau)] .
\end{aligned}
$$

(See Ref. [16].) It should be noted that $y_{t}^{0}$ is a deterministic, rather than a stochastic, process. 
Then, we can express $\mathcal{D}$ in terms of $y_{t}^{0}$ as

$$
\mathcal{D}=\lim _{T \rightarrow \infty} \frac{D^{2}}{T} \int_{0}^{T}\left(y_{s}^{0}\right)^{2} d s .
$$

Moreover, we examined how the function $y_{t}^{0}$ behaves in our numerical computations of Eq. (11), and found that it seemed to converge to a constant of $\bar{y} \propto \frac{1}{1+K \tau}$, as described in the Appendix and in Ref. [17]. As a consequence, $\mathcal{D}$ is given by

$$
\mathcal{D}=(D \bar{y})^{2} .
$$

In addition, it should be noted that this $\bar{y}$ and $\mathcal{D}$ decrease with increasing $\tau$, which is consistent with Fig. 2.

Here, the characteristics of our model should be stressed. In Ref. [16], the equivalent conditions to stationary solutions of $y=\left\{y_{t}\right\}$ are given (see the definition of the stationarity in the Appendix), which are not met in our system (10). In the case of the stationary $y$, its diffusion coefficient $\mathcal{D}$ goes to zero as $T \rightarrow \infty$.

This can be seen in another intuitive way. If a limiting process of our model (9) is considered with $\tau \rightarrow 0$,

$$
d y_{t}^{\downarrow}=D d w_{t} .
$$

It should be noted that this is just a scaled random walk, which still includes $\mathcal{D}=D^{2}$. On the other hand, $\mathcal{D}=0$, under another limiting process with $\tau \rightarrow \infty$,

$$
d y_{t}^{\uparrow}=K\left(y_{-\infty}-y_{t}^{\uparrow}\right) d t+D d w_{t},
$$

where $y_{-\infty}:=\lim _{t \rightarrow-\infty} y_{t}$ is given as an initial condition and as a constant target control level.

As derived before, in our model, the diffusion coefficient is given by $\mathcal{D}=D^{2}\left(\frac{1}{1+K \tau}\right)^{2}$. Thus, any level of $\mathcal{D}$ between $\left(0, D^{2}\right]$ can be achieved with an appropriate choice of $K$ and $\tau$.

\section{APPLICATION TO MOLECULAR DYNAMICS}

In the following, we apply the control scheme which is shown above to a molecular dynamics model.

We consider the dynamics of particles with noise, and attractive and dissipative forces in a 1D space,

$$
\begin{aligned}
& \frac{d x_{i}}{d t}=v_{i}, \\
& \frac{d v_{i}}{d t}=P_{i}\left(\left\{x_{j}\right\}_{j=1}^{N}\right)+D_{i}\left(\left\{v_{j}\right\}_{j=1}^{N}\right)+R \xi,
\end{aligned}
$$

where $x_{i}$ and $v_{i}$ are the position and velocity of the $i$ th particle, $\xi$ is noise from the Gaussian distribution with intensity $R$, and $N$ is the number of particles. For simplicity, we assume that the mass of the $i$ th particles, $m_{i}=1$. The first term in Eq. (14) is a conservative force derived as a derivative of the potential $U$ written as

$$
\begin{aligned}
P_{i} & =-\frac{d U\left(\left\{x_{j}\right\}_{j=1}^{N}\right)}{d x_{i}}, \\
U\left(\left\{x_{j}\right\}_{j=1}^{N}\right) & =\sum_{i=1}^{N} \sum_{j=i+1}^{N} \tilde{V}\left(x_{i j}\right),
\end{aligned}
$$

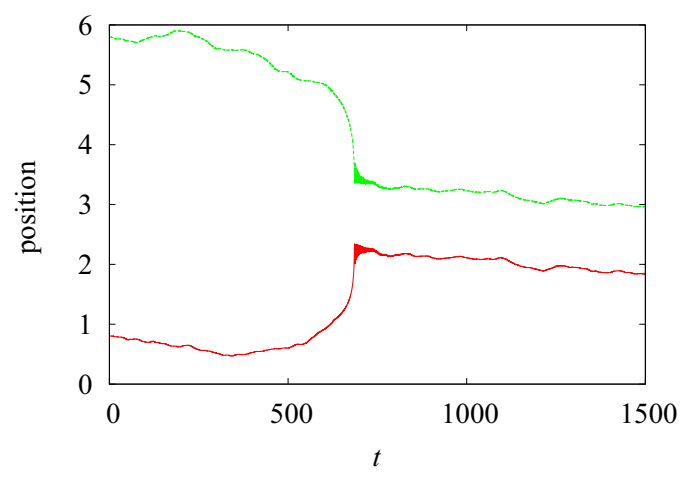

FIG. 3. Time evolutions of the positions of two particles without control inputs. Particles flocculate because of the interacting force, and move together diffusively.

where $\tilde{V}$ is a central force potential, and $x_{i j}=x_{i}-x_{j}$. In this paper, we use the Lennard-Jones potential as the central potential as given by

$$
\tilde{V}(x)=\epsilon\left[\left(\frac{\sigma}{x}\right)^{12}-\left(\frac{\sigma}{x}\right)^{6}\right] .
$$

The second term in Eq. (14) represents a dissipative force,

$$
D_{i}=-\gamma v_{i},
$$

where $\gamma$ is a coefficient of drag. We set $\sigma=2^{-1 / 6}$ so that the LJ potential $\tilde{V}(x)$ has a minimum at $x=1$ and $\epsilon=1$.

We calculate the dynamics of two particles $(N=2)$ with initial conditions $x_{1}=0.8, v_{1}=0.0, x_{2}=5.8$, and $v_{2}=0.0$. It should be noted that other values of the initial conditions provide similar results below. In this case, the particles flocculate because of the attracting force from the LJ potential, and move together diffusively (see Fig. 3).

In this application, we control fluctuations in the system by using the time-delayed feedback control method. Velocities fluctuate randomly around 0 because of the Gaussian noise and are stable at 0 without noise. Thus, the dynamics of the velocities is the same as the Gaussian noise. This Gaussian noiselike behavior makes the dynamics of the positions analogous to a simple random walk, $\frac{d x_{i}}{d t} \sim \xi$. Thus, control inputs are given only according to the dynamics of the positions, which are driven by noise originally from the velocities. The control scheme for the molecular dynamics model is written as

$$
\begin{aligned}
& \frac{d x_{i}}{d t}=v_{i}-K\left[x_{i}-x_{i}(t-\tau)\right], \\
& \frac{d v_{i}}{d t}=P_{i}\left(\left\{x_{j}\right\}_{j=1}^{N}\right)+D_{i}\left(\left\{v_{j}\right\}_{j=1}^{N}\right)+R \xi .
\end{aligned}
$$

Control signals are injected when the particles are flocculated. Figure 4(a) shows the dynamics of the center positions of two particles $(N=2)$ without control (dotted line) and with control with $\tau=10$ (solid line). In the case of three particles $(N=3)$, the dynamics of the center positions without control and with control is shown in Fig. 4(b). The movement of the flocculated particles is diffusive because of noise. These figures show that the diffusion of the flocculated particles is suppressed by our control method. 

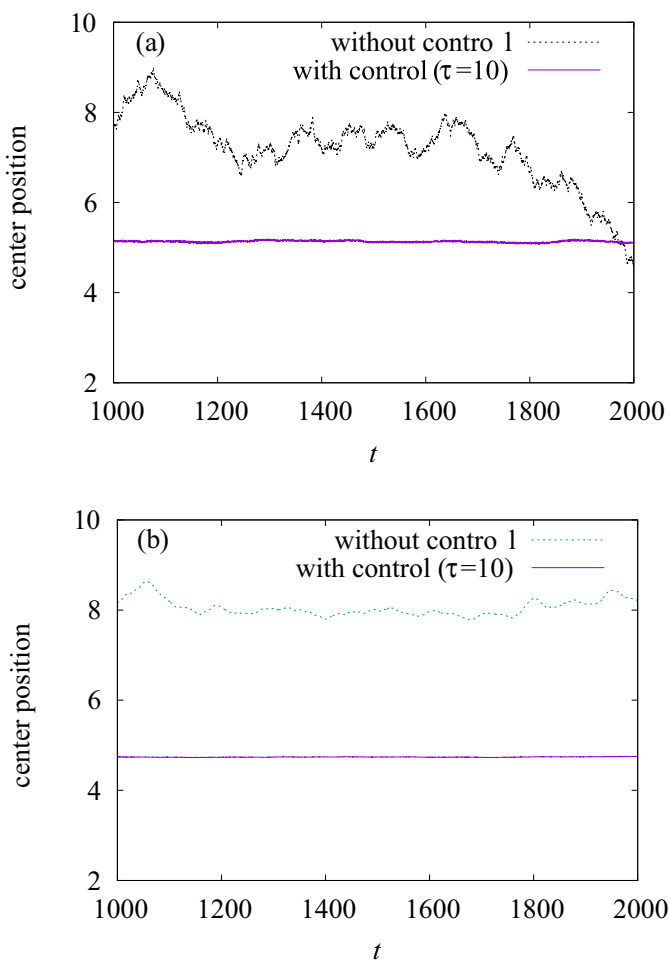

FIG. 4. Time evolutions of the center position of (a) two particles and (b) three particles. The green and the red lines are the time evolutions without control inputs and with control input $(\tau=10)$, respectively.

Figure 5 shows the dependence of the $\mathcal{D}$ with $\tau$. As is the case with the simple random walk, the $\mathcal{D}$ decreases with a change in $\tau$.

\section{DISCUSSION}

In this paper, we have applied time-delayed feedback control to a 1D random walk model. The interaction between noise and time delay is nontrivial. We have observed that the diffusion coefficient decreases with increasing delay time. Thus, we have analytically explained the decay of the diffusion coefficient by solving the stochastic delay differential equations derived from the controlled system. In principle, it

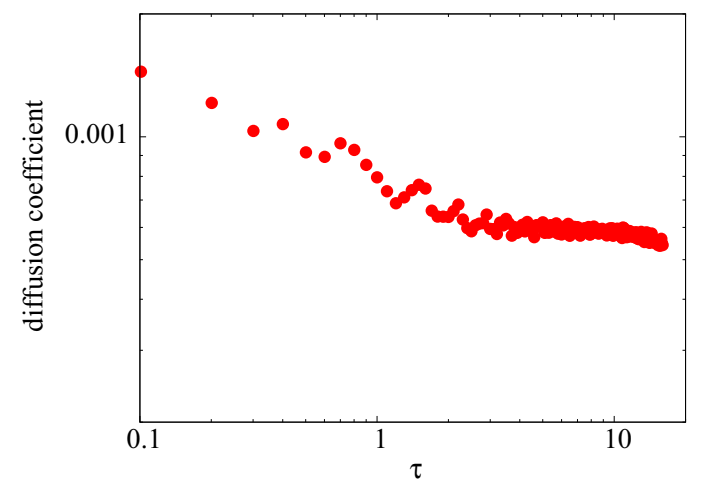

FIG. 5. The diffusion coefficient $\mathcal{D}$ of the molecular dynamics model with two particles as a function of $\tau$. is possible to control the amount of diffusion from zero to a specific value.

We have applied the proposed method to a molecular dynamics model described by continuous time differential equations. We have numerically demonstrated the control of two flocculated particles with the LJ potential. Consequently, diffusion generated by noise was successfully controlled and decay of the diffusion coefficient was observed in this system.

The proposed method does not change the physical properties of the system in comparison to the conventional technique of reducing diffusion, i.e., decreasing temperature, which can change the thermophysical properties. Moreover, the previous analysis of delayed random walks is based on the assumption of stationarity $[14,18]$. In contrast, our method is not stationary and a conventional analysis by stochastic delay differential equations in Ref. [16] cannot be applied. In addition to such theoretical findings, we remark that the method can be applied to nanomicroscopic resonators for reducing thermal noise. In those experimental systems, time delay cannot be avoided or shortened less than some length $[11,12]$. However, making a longer time delay can be easy so that our method is effective in the systems.

Other types of time-delayed feedback control are also feasible. For example, we have proposed the adaptive time-delayed system as follows [19], $x_{n+1}=x_{n}+D \xi_{n}-1 / x_{n-\tau}\left(x_{n}-\right.$ $\left.x_{n-\tau}\right)$. This form of adaptive time-delayed feedback control shows almost similar performance to the original system (2) in terms of control of the diffusion coefficient. The difference between the original and the adaptive control is in determining $K$ or determining the initial values for the system. We can apply the adaptive control scheme to real world systems depending on the availability of the control parameters. If we cannot access $K$, adaptive feedback gain $1 / x_{n-\tau}$ can be alternatively used.

\section{ACKNOWLEDGMENTS}

In this work, we used the computer of the MEXT Joint Usage/Research Center "Center for Mathematical Modeling and Applications," Meiji University, Meiji Institute for Advanced Study of Mathematical Sciences (MIMS). The authors thank JSPS Grants-in-Aid No. 15K12137 and 15K17582 for support. H.A. thanks Council for Science, Technology and Innovation (CSTI), Cross-Ministerial Strategic Innovation Promotion Program (SIP), Next-Generation Power Electronics (NEDO) for support.

\section{APPENDIX : SOLVING SDDE}

\section{Derivation of the diffusion coefficient $\mathcal{D}$}

Here, we examine the following SDE with delay (SDDE),

$$
d y_{t}^{(\tau)}=K\left(y_{t-\tau}^{(\tau)}-y_{t}^{(\tau)}\right) d t+D d w_{t}, \quad t \geqslant 0,
$$

where $\left\{w_{t}\right\}_{0 \leqslant t \leqslant T}$ is a standard Wiener process on a filtered probability space $\left(\Omega, \mathcal{F},\left\{\mathcal{F}_{t}\right\}, P\right), K$ is a positive constant representing the speed of "mean reversion" to a delayed control $y_{t-\tau}^{(\tau)}, D$ is a positive constant for a volatility of $y_{t}^{(\tau)}$, and $\tau$ is the delay interval for the control. $y_{s}^{(\tau)}$ for $s \in[\tau, 0]$ is assumed to be a given $\mathcal{F}_{0}$-measurable random variable $Z_{s}$. 
In the remainder of this section, we derive the concrete expression for its diffusion coefficients

$$
\mathcal{D}:=\lim _{T \rightarrow \infty} \frac{V\left[y_{T}^{(\tau)}\right]}{T},
$$

where $V[x]$ is a variance of $x$. Owing to the work by Kuchler and Mensch [16], we can construct the unique solution to the $\operatorname{SDDE}(\mathrm{A} 1)$ as

$$
\begin{aligned}
& y_{t}^{(\tau)}=y_{t}^{Z}+D \int_{0}^{t} y_{t-s}^{0} d w_{s}, \quad 0 \leqslant t, \\
& y_{t}^{(\tau)}=Z_{t}, \quad-\tau \leqslant t \leqslant 0,
\end{aligned}
$$

where

$$
\begin{aligned}
y_{t}^{Z}: & =y_{t}^{0} Z_{0}+K \int_{-\tau}^{0} y_{t-s-\tau}^{0} Z_{s} d s, \\
y_{t}^{0}: & =\sum_{n=0}^{\lceil t / \tau\rceil}\left(\frac{K^{n}}{n !}\right)(t-n \tau)^{n} \exp [-K(t-n \tau)] .
\end{aligned}
$$

(For details, see Proposition 2.2 in Ref. [16].)

It should be stressed that $y_{t}^{0}$ is a deterministic, nonstochastic process, and the first term on the right-hand side of (A2), that is, $y_{t}^{Z}$, is a random process whose outcomes are available with the filtration $\mathcal{F}_{0}$, the information at $t=0$. In contrast, the second term in (A2) is independent from $\mathcal{F}_{0}$ and hence from $y_{t}^{Z}$.

With this solution to $y^{(\tau)}$, we first compute a conditional variance of $y_{T}^{\tau}$ on the initial filtration $\mathcal{F}_{0}$.

Proposition 1. The expectation and variance of $y_{T}^{(\tau)}$ conditional on $\mathcal{F}_{0}$ is given by

$$
E\left[y_{T}^{(\tau)} \mid \mathcal{F}_{0}\right]=y_{T}^{Z}
$$

and

$$
\begin{aligned}
V\left[y_{T}^{(\tau)} \mid \mathcal{F}_{0}\right] & =V\left[y_{T}^{Z} \mid \mathcal{F}_{0}\right]+V\left[D \int_{0}^{T} y_{T-s}^{0} d w_{s} \mid \mathcal{F}_{0}\right] \\
& =D^{2} \int_{0}^{T}\left(y_{T-s}^{0}\right)^{2} d s .
\end{aligned}
$$

Proof. The expectation is directly derived from (A2) and the martingale property of stochastic integrals. For the variance, the first line is derived from the independence between $y_{T}^{Z}$ and $\int_{0}^{T} y_{T-s}^{0} d w_{s}$, and then is derived from Itô's isometry and availability of $y_{T}^{Z}$ with $\mathcal{F}_{0}$.

Then, a total (unconditional) variance of $y_{T}^{(\tau)}$ without the information about the outcomes of $Z_{s}$ for $s \leqslant 0$ is computed as

$$
\begin{aligned}
V\left[y_{T}^{(\tau)}\right] & =E\left[\left(y_{T}^{(\tau)}\right)^{2}\right]-E\left[y_{T}^{(\tau)}\right]^{2} \\
& =E\left[E\left[\left(y_{T}^{(\tau)}\right)^{2} \mid \mathcal{F}_{0}\right]\right]-E\left[E\left[y_{T}^{(\tau)} \mid \mathcal{F}_{0}\right]\right]^{2} \\
& =E\left[V\left[y_{T}^{(\tau)} \mid \mathcal{F}_{0}\right]+E\left[y_{T}^{(\tau)} \mid \mathcal{F}_{0}\right]^{2}\right]-E\left[E\left[y_{T}^{(\tau)} \mid \mathcal{F}_{0}\right]\right]^{2} \\
& =E\left[D^{2} \int_{0}^{T}\left(y_{T-s}^{0}\right)^{2} d s+\left(y_{T}^{Z}\right)^{2}\right]-E\left[y_{T}^{Z}\right]^{2} \\
& =D^{2} \int_{0}^{T}\left(y_{T-s}^{0}\right)^{2} d s+E\left[\left(y_{T}^{Z}\right)^{2}\right]-E\left[y_{T}^{Z}\right]^{2} .
\end{aligned}
$$

First, since $y_{t}^{0}$ is a deterministic function of $t$, as mentioned earlier, we have

$$
E\left[y_{T}^{Z}\right]=y_{T}^{0} E\left[Z_{0}\right]+K \int_{-\tau}^{0} y_{T-s-\tau}^{0} E\left[Z_{s}\right] d s .
$$

As described later, the function $y_{t}^{0}$ seems to converge to a certain level $\bar{y}$; thus, the last term on the right-hand side of Eq. (A5) actually contributes nothing to the diffusion coefficient $\mathcal{D}$ because of the division by $T$.

Similarly, the second term on the right-hand side of Eq. (A5), that is,

$$
\begin{aligned}
E\left[\left(y_{T}^{Z}\right)^{2}\right]= & E\left[\left(y_{T}^{0} Z_{0}\right)^{2}+2\left(y_{T}^{0} Z_{0}\right)\left(K \int_{-\tau}^{0} y_{T-s-\tau}^{0} Z_{s} d s\right)\right. \\
& \left.+\left(K \int_{-\tau}^{0} y_{T-s-\tau}^{0} Z_{s} d s\right)^{2}\right] \\
= & \left(y_{T}^{0}\right)^{2} E\left[Z_{0}^{2}\right]+2 K y_{T}^{0} E\left[Z_{0} \int_{-\tau}^{0} y_{T-s-\tau}^{0} Z_{s} d s\right] \\
& +K^{2} E\left[\left(\int_{-\tau}^{0} y_{T-s-\tau}^{0} Z_{s} d s\right)^{2}\right],
\end{aligned}
$$

again has no contribution to $\mathcal{D}$ because it disappears when $T$ becomes infinite.

Thus, we can conclude that the diffusion coefficient $\mathcal{D}$ of $y^{(\tau)}$ is given by

$$
\mathcal{D}=\lim _{T \rightarrow \infty} \frac{1}{T} D^{2} \int_{0}^{T}\left(y_{T-s}^{0}\right)^{2} d s,
$$

that is, an "average over time" of $\left(y_{T-s}^{0}\right)^{2}$. From this equation, we can estimate that a change in $D_{y}^{(\tau)}$ due to a change of $\tau$ is strongly dependent on the behavior of the fundamental solution $y_{t}^{0}$ when $\tau$ moves.

\section{Behavior of $y_{t}^{0}$}

In this section, we examine the behavior of $y_{t}^{0}$, which plays a very important role in the behavior of $\mathcal{D}$.

First, we mention the relation between the behavior and stationarity of $y^{(\tau)}$. In Ref. [16], the authors proved several equivalent conditions for the stationarity of the solution to a SDDE, for example,

$$
d x_{t}=\left(a x_{t}+b x_{t-\tau}\right) d t+D d w_{t},
$$

which is a generalization of our model (A2) (in our model, $a=-K$ and $b=K)$. One of those conditions is a negativity of $v_{0}(a, b, \tau)$, and

$$
v_{0}(a, b, \tau)=\max \{\operatorname{Re}(\lambda) ; h(\lambda)=0\},
$$

where $h(\lambda)$ is a characteristic function given by

$$
h(\lambda)=\lambda-a-b \exp (-\lambda \tau),
$$

and $\operatorname{Re}(x)$ is a real part of $x \in \mathbf{C}$.

Unfortunately, it is easily shown that $v_{0}(-K, K, \tau)=0$, indicating a nonstationary solution in our model.

Proposition 2. Considering our model (A2) with $a=-K$, and $b=K, v_{0}(-K, K, \tau)=0$.

Proof. First, it is obvious that $\lambda=0$ solves $h(\lambda)=0$. 
Second, assume a characteristic root of $h(\lambda)=0$ with the form $z=\epsilon+i \beta$, where $\epsilon>0$ and $i^{2}=-1$. Then,

$$
\begin{aligned}
\operatorname{Re}[h(z)] & =\operatorname{Re}\{(\epsilon+i \beta)+K-K \exp [-(\epsilon+i \beta) \tau]\} \\
& =\epsilon+K-K \exp (-\epsilon \tau) \cos \beta \tau \\
& \geqslant \epsilon+K[1-\exp (-\epsilon \tau)]>0 .
\end{aligned}
$$

There exists a contradiction to the assumption that $z$ is the root of $h(z)=0$, leading us to the conclusion that $v_{0}(-K, K, \tau)=0$.

Moreover, in Proposition 3.2 in Ref. [16], $y_{t}^{0}$, which is the fundamental solution to (A3), was proved to be strictly positive on $(0, \infty)$ and

$$
\lim _{t \rightarrow \infty} t^{-1} \ln y_{t}^{0}=v_{0}(-K, K, \tau) .
$$

Thus, the aforementioned findings show that our $y_{t}^{0}$ does not grow positively or negatively faster than $t$.
Moreover, although not yet proved in a rigorous manner, with our several numerical computations of the constructive definition (A3), $y_{t}^{0}$ is confirmed to almost converge to the constant $\bar{y}=\frac{1}{1+K \tau}$, which is decaying in $\tau$. The rigorous proof of the relation $\bar{y}=\frac{1}{1+K \tau}$ should be explored by comparing the coefficients of the expanded $\frac{1}{1+K \tau}$ in a series with those of Eq. (11) at $t=N \tau$, $N=\{1,2,3, \ldots\}$.

Thus, $\mathcal{D}$ is eventually given by

$$
\mathcal{D}=\lim _{T \rightarrow \infty} \frac{1}{T} D^{2} \int_{0}^{T}\left(y_{T-s}^{0}\right) d s=(D \bar{y})^{2},
$$

with the same decaying pattern, which is consistent with Fig. 2.
[1] A. Einstein, Ann. Phys. 322, 549 (1905).

[2] Z. Siwy and A. Fulinski, Phys. Rev. Lett. 89, 198103 (2002).

[3] D. Arcizet, B. Meier, E. Sackmann, J. O. Rädler, and D. Heinrich, Phys. Rev. Lett. 101, 248103 (2008).

[4] B. I. Shraiman and E. D. Siggia, Nature (London) 405, 639 (2000).

[5] J. J. Collins and C. J. De Luca, Exp. Brain Res. 95, 308 (1993).

[6] T. Fujita et al., Nano Lett. 14, 1172 (2014).

[7] V. Tabard-Cossa et al., Nanotechnology 18, 305505 (2007).

[8] H. J. Butt and M. Jaschke, Nanotechnology 6, 1 (1995).

[9] K. Murali, S. Sinha, W. L. Ditto, and A. R. Bulsara, Phys. Rev. Lett. 102, 104101 (2009).

[10] M. Igarashi, C.-H. Huang, T. Morie, and S. Samukawa, Appl. Phys. Express 3, 085202 (2010).

[11] M. Bonaldi, L. Conti, P. De Gregorio, L. Rondoni, G. Vedovato, A. Vinante, M. Bignotto, M. Cerdonio, P. Falferi, N. Liguori,
S. Longo, R. Mezzena, A. Ortolan, G. A. Prodi, F. Salemi, L. Taffarello, S. Vitale, and J.-P. Zendri, Phys. Rev. Lett. 103, 010601 (2009).

[12] T. Munakata and M. L. Rosinberg, Phys. Rev. Lett. 112, 180601 (2014)

[13] K. Pyragas, Phys. Lett. A 170, 421 (1992).

[14] T. Ohira and J. G. Milton, Phys. Rev. E 52, 3277 (1995).

[15] A. G. Balanov, N. B. Janson, and E. Schöll, Physica D 199, 1 (2004).

[16] U. Küchler and B. Mensch, Stochastics Stochastic Rep. 40, 23 (1992).

[17] M. U. Kobayashi et al. (unpublished).

[18] T. Ohira and T. Yamane, Phys. Rev. E 61, 1247 (2000).

[19] H. Ando and M. U. Kobayashi, IFAC-PapersOnLine 48, 262 (2015). 